\title{
Characterization of Initial Stages of Diabetic Macular Edema
}

\author{
Ana Rita Santos ${ }^{a, b}$ Torcato Santos ${ }^{a}$ Dalila Alves ${ }^{a}$ Inês P. Marques ${ }^{a}$ \\ Conceição Lobo ${ }^{a, c}$ José Cunha-Vaz ${ }^{a, c}$ \\ ${ }^{a}$ AIBILI, Association for Innovation and Biomedical Research on Light and Image, Coimbra, Portugal; \\ ${ }^{b}$ Department of Orthoptics, Superior School of Health, Polytechnic of Porto, Porto, Portugal; \\ 'University of Coimbra, Coimbra, Portugal
}

\section{Keywords}

Diabetic retinopathy Macular edema - Optical coherence tomography - Optical coherence tomography-leakage

\begin{abstract}
Purpose: This study is aimed at characterizing the type of retinal edema in the initial stages of retinopathy in type 2 diabetes. Methods: In this retrospective cross-sectional study, spectral domain optical coherence tomography (OCT) layer by layer analysis of the retina in association with OCTLeakage, an algorithm to detect sites of low optical reflectivity, were used to examine eyes with minimal, mild, and moderate diabetic retinopathy (DR). Results: A total of 142 eyes from 142 patients ( $28 \%$ women) aged $52-88$ years were imaged. Macular edema, either subclinical (SCME) or centralinvolved macular edema (CIME), was present in $43 \%$ of eyes in group $10-20,41 \%$ of eyes in group 35 , and $38 \%$ of eyes in group 43-47. The inner nuclear layer (INL) was the layer showing higher and most frequent increases in retinal thickness (79\%). The edema was predominantly intracellular in group 10-20 (65\%) and extracellular in groups 35 (77\%) and 43-47 (69\%). Conclusions: Eyes from diabetic patients in the initial stages of DR with different Early Treatment Diabetic Retinopathy Study gradings show similar prevalence of SCME and CIME, independent of the severity of the retinopa-
\end{abstract}

thy. Retinal edema is located mainly in the INL and appears to be mostly extracellular except in the earliest stages of diabetic retinal disease where intracellular edema predominates.

(c) 2019 S. Karger AG, Basel

\section{Introduction}

Retinal edema is the most frequent cause of vision loss due to diabetes [1]. Its characterization and understanding are fundamental for its prevention, and for developing novel targeted treatments. Optical coherence tomography (OCT) allows objective and quantitative evaluation of diabetic macular edema, providing cross-sectional images of the internal microstructures of the retina and reproducible thickness measurements [2].

OCT has improved the investigator's ability to follow macular edema changes, allowing the detection of increased retinal thickness (RT), that is, edema, even before clinical detection by slit-lamp examination [3]. The term subclinical macular edema (SCME) has been proposed to describe these early stages of macular edema [4, 5]. There have been reports showing that the increase in RT in type 2 diabetes occurs mainly in the inner nuclear layer (INL), either due to leakage from the deep retinal

\section{KARGER}

(c) 2019 S. Karger AG, Basel

E-Mail karger@karger.com

www.karger.com/ore
José Cunha-Vaz

AIBILI, Azinhaga de Santa Comba

Celas

PT-3000-548 Coimbra (Portugal)

E-Mail cunhavaz@ aibili.pt 
plexus (DRP) [6], or associated with Müller cell swelling [7]. We have reported an algorithm designated OCTLeakage that is capable of performing automated analysis of changes in the retinal extracellular space by identifying sites of lower optical reflectivity. Our group has shown, using OCT-Leakage that it is possible to identify in the full retina and in its different layers, the location and extent of the sites of extracellular edema in the retina $[8,9]$.

For this study, we examined, with spectral domain OCT (SD-OCT) and the OCT-Leakage algorithm, eyes in the initial stages of diabetic retinopathy (DR), well identified by the Early Treatment Diabetic Retinopathy Study (ETDRS) severity scale. The aim of the study was to identify, in the initial stages of retinopathy, the rates of occurrence of macular edema and the relative contribution of the intracellular and extracellular components in diabetic retinal edema.

\section{Research Design and Methods}

In this retrospective cross-sectional study of data from an observational study (ClinicalTrials.gov identifier: NCT03010397), participants with diabetes in the initial stages of non-proliferative diabetic retinopathy (NPDR) were included from February 2016 to April 2018. The tenets of the Declaration of Helsinki were followed, and approval was obtained from the Institutional Ethical Review Board. Written informed consent to participate in the study was obtained from all individuals after all procedures were explained. All participants underwent a full ophthalmological examination, SD-OCT and OCT angiography (OCTA) imaging using CIRRUS ${ }^{\mathrm{TM}}$ HD-OCT 5000 with AngioPlex ${ }^{\circledR}$ OCTA (Carl Zeiss Meditec, Dublin, CA, USA), and color fundus photography. A total of 142 eyes from 142 patients with diabetes, one eye per patient, were classified into 3 groups according to the ETDRS severity level: no or minimal NPDR, ETDRS levels 10-20 (54 eyes); mild NPDR, ETDRS level 35 (54 eyes); and moderate NPDR, ETDRS levels 43-47 (34 eyes). The DR severity level was determined by 2 independent graders within the context of an experienced reading centre and was based on the 7-field protocol using the ETDRS classification. The eye showing the more advanced ETDRS grading in any given patient was chosen to be the study eye.

Age, duration of diabetes, and hemoglobin $\mathrm{A}_{1 \mathrm{C}}(\mathrm{HbAlc})$ level, as an indicator of diabetic metabolic control, were collected for each participant from their patient records. Visual acuity was measured for each eye using the ETDRS protocol and Precision Vision charts at 4 meters.

Exclusion criteria included any previous laser treatment or intravitreal injections, or presence of age-related macular degeneration, glaucoma, or vitreomacular disease and high ametropia (spherical equivalent greater than -6 and +2 DPT), or any other systemic disease that could affect the eye, with special attention to uncontrolled systemic hypertension (values outside normal range: systolic $70-210 \mathrm{mmHg}$ and diastolic $50-120 \mathrm{mmHg}$ ) and history of ischemic heart disease. The information about previous medical conditions and ocular treatments was obtained by interviewing each participant.

A control group of 106 eyes from healthy controls (36 women and 25 men; mean [Standard Deviation (SD)] age, 47.6 [12.8] years) was used to compare with the study group.

Images were acquired using the Cirrus AngioPlex instrument. This SD-OCT instrument, now capable of scanning at a rate of 68,000 A-scans per second, continues to use a superluminescent diode (centre wavelength, $840 \mathrm{~nm}$; bandwidth, $90 \mathrm{~nm}$ ) as its optical source, with an A-scan depth of $2.0 \mathrm{~mm}$ (1024 pixels), axial resolution of $5 \mu \mathrm{m}$ in tissue, and a transverse resolution of $15 \mu \mathrm{m}$. The AngioPlex OCTA is capable of non-invasive depth-encoded imaging of the retinal and choroidal microvasculature. It also incorporates FastTrac retinal-tracking technology to achieve 3-dimensional en-face angiographic images with minimal movement artifacts. All scans acquired using AngioPlex imaging can also be viewed using the standard intensity-based en-face algorithms available on the Cirrus.

\section{Thickening of the Retina Layers (Edema)}

The Macular Cube $512 \times 128$ acquisition protocol, consisting of $128 \mathrm{~B}$-scans with $512 \mathrm{~A}$-scans each, was used to assess the subjects' central RT (CRT) by using the standard Cirrus examination reports.

Segmentation of retinal layers to determine thickness values in the central subfield, was performed on the structural OCTA data using a segmentation software implemented by AIBILI [10]. Segmentation results were reviewed by a masked grader, and corrections were performed when needed.

None of the eyes in this series of patients were observed to have cystoid macular edema of the retina or disorganization of the retinal inner layers (DRIL) that could have influenced the segmentation analysis. Presence of retinal cystoid changes was defined as hyporeflective empty spaces above 8 voxels in retinal layers [11, 12]. DRIL was defined as the loss of boundaries between the ganglion cell layer and inner plexiform layer complex, INL and outer plexiform layer (OPL) $[13,14]$.

Eyes with subclinical and clinical macular edema (central-involved macular edema [CIME]) were identified following the reference values established by the DRCR.net for Cirrus SD-OCT [5, 15]. CIME is defined as CRT $\geq 290 \mu \mathrm{m}$ in women and $\geq 305 \mu \mathrm{m}$ in men; SCME is defined as CRT between 260 and $290 \mu \mathrm{m}$ in women and between 275 and $305 \mu \mathrm{m}$ in men.

\section{OCT-Leakage Measurements}

Because the noise and signal levels in the AngioPlex scan (protocol Angiography $6 \times 6 \mathrm{~mm}$ ) are different from the noise and signal levels on the Macular Cube $512 \times 128$ scan used in the original OCT-Leakage method [16], the threshold needs to be set differently. Data on 41 normal eyes were collected to set an appropriate threshold of low optical reflectivity (LOR) for the AngioPlex Angiography $6 \times 6$ - $\mathrm{mm}$ scan.

Sites of LOR are depicted as 2-dimensional images of the area of the retina by assigning a single representative value for each Ascan, which represents the existence of reflectivity values less than a predefined threshold for optical reflectivity from A-scans obtained from a healthy control population [16]. The white areas depicted in the LOR maps represent the locations of A-scans having reflectivity values less than the predefined threshold; otherwise 
locations appear as black areas. Extracellular fluid distribution of a given area of the retina can be measured by the LOR area ratio, which stands for the fraction of the number of A-scans with optical reflectivity values less than the threshold and the total number of A-scans within the considered area. LOR sites were identified in the different retinal layers. Maps of LOR sites are therefore obtained not only for the full retina, but also layer by layer as en-face images.

To categorize changes in LOR ratio, they were considered absent if the values were within normal range of the healthy control group, "possible" if different by more than $1 \mathrm{SD}$, and "definite" if different by more than 2 SD. Eyes with increased RT (1 or 2 SD) without corresponding increase in LOR ratio ( 1 or 2 SD) were considered to have intracellular edema whereas eyes with increased $\mathrm{RT}$ with corresponding increases in LOR ratios (1 SD or $2 \mathrm{SD}$ ) were considered to have extracellular edema.

\section{Capillary Dropout}

OCTA data were collected with the AngioPlex device using the Angiography $3 \times 3 \mathrm{~mm}$ acquisition protocol. This acquisition protocol scans a $3 \times 3 \times 2 \mathrm{~mm}^{3}$ volume in the central macula, consisting of a set of 245 clusters of B-scans repeated 4 times, where each B-scan consists of 245 A-scans. The AngioPlex eye tracking algorithm was used to reduce the effect of eye motion artifacts. Images were evaluated at the time of acquisition for quality, as signal strength $>7$ was required, with minimal motion artifacts and no evidence of defocus or blur. Only images that passed these quality checks were considered.

Vessel density metrics for the entire $3 \times 3 \mathrm{~mm}^{2}$ central macular area were computed for the superficial retinal plexus (SRP) and DRP by the automated Carl Zeiss Density Exerciser software (version 10.0.0.12787) [17].

Changes in retinal vessel density were considered "absent" if the values were within the normal range of the healthy control groups, "possible" if different by more than 1 SD, and "definite" if different from normal values by more than $2 \mathrm{SD}$.

\section{Statistical Analysis}

Reference values were taken considering the mean and SD values of the healthy control population taking the patient's gender into consideration.

Differences from the control population adjusted by gender were categorized by ETDRS severity groups using the mean and SD. For each metric, the number of alterations over the mean plus (minus) 2 SD related to the normal healthy control population was counted and the corresponding 95\% Confidence Interval (CI) was computed using the Clopper-Pearson method.

To compare the differences from the healthy control population between patients with normal RT, SCME and CIME the Kruskal-Wallis non-parametric test was used.

Comparison of the ETDRS groups for categorical outcomes was conducted using the Chi-square test.

To explore correlations, the Pearson coefficient and the respective significance were computed.

Multivariate logistic regression of the study outcomes was performed (adjusted for baseline characteristics) to compare the $3 \mathrm{ET}$ DRS groups.

Statistical analysis was performed with Stata 12.1 (Stata Corp. LP, College Station, TX, USA), and a $p$ value $\leq 0.05$ was considered to be statistically significant.

Initial Stages of Diabetic Macular Edema

\section{Results}

A total of 142 eyes from 142 patients (28\% women) aged $52-88$ years were imaged. The distribution of characteristics of eyes/patients for each of the stages of the disease is described by Marques et al. [17]. No statistically significant differences were found between eyes/patients within different stages of the disease, except for hemoglobin $\mathrm{A}_{1 \mathrm{C}}$ levels that reflected an association between poorer metabolic control and more severe stages of the disease.

\section{Retinal Thickening (Edema) and LOR Ratios}

Increases in RT of the full retina reached SCME levels in 19 of 54 eyes graded ETDRS 10-20 (35\%, [95\% CI $22-48 \%$ ]), in 18 of 54 eyes graded ETDRS 35 (33\%, [ $95 \%$ CI $21-46 \%]$ ), and in 9 of 34 eyes graded $43-47$ (26\%, [95\% CI 12-41\%]). CIME was found in 4 of 54 eyes graded ETDRS $10-20$ (7\%, [95\% CI $0.4-4 \%])$, in 4 of 54 eyes graded ETDRS 35 (7\%, [95\% CI 0.4-4\%]), and in 4 of 34 eyes graded ETDRS $43-47$ (12\%, [95\% CI 0.9$23 \%]$ ).

When comparing RT values obtained from different segmented retinal layers - retinal nerve fiber layer, ganglion cell layer and inner plexiform layer, INL, OPL, outer nuclear layer and inner segment (ONL+IS), outer segment, retinal pigment epithelium - in eyes/patients with SCME and CIME with eyes/patients with normal overall $\mathrm{RT}$ and with age-matched non-diabetic control eyes, selective involvement of specific retinal layers is clearly apparent (Fig. 1).

In the eyes with increased RT, the INL is the layer showing higher and most frequent increases in RT (79\%). There were also increases in the ONL+IS and in the OPL but less frequently (57 and $41 \%$, respectively).

Multivariate analysis showed correlation of retinal edema with metabolic control $(p=0.007)$ but not with severity of the retinopathy, as identified by ETDRS grading, age, duration of disease or visual acuity.

Eyes presenting macular edema, either SCME or CIME, show increase in LOR ratios, involving mainly the INL and the OPL. The increases in RT were significantly correlated with increases in the LOR ratios (INL: $r=0.45$, $p<0.001$; OPL $=0.48, p<0.001$ ).

Increased LOR ratios in the ONL+IS only occurred in eyes where there were increases in the INL or in the OPL (online suppl. Tables 1-3; for all online suppl. material, see www.kager.com/doi/10.1159/000499117).

In the presence of increased RT, either SCME or CIME, there were differences in the identification of sites of low- 
Fig. 1. Mean retinal layer thickness difference $(\mu \mathrm{m})$ and 95\% CI between eyes/patients with normal RT, SCME and CIME, in the central subfield. SCME, subclinical macular edema; CIME, central-involved macular edema; RT, retinal thickness; RNFL, retinal nerve fiber layer; GCL+IPL, ganglion cell layer and inner plexiform layer; INL, inner nuclear layer; OPL, outer plexiform layer; ONL+IS, outer nuclear layer and inner segment; OS, outer segment; RPE, retinal pigment epithelium.

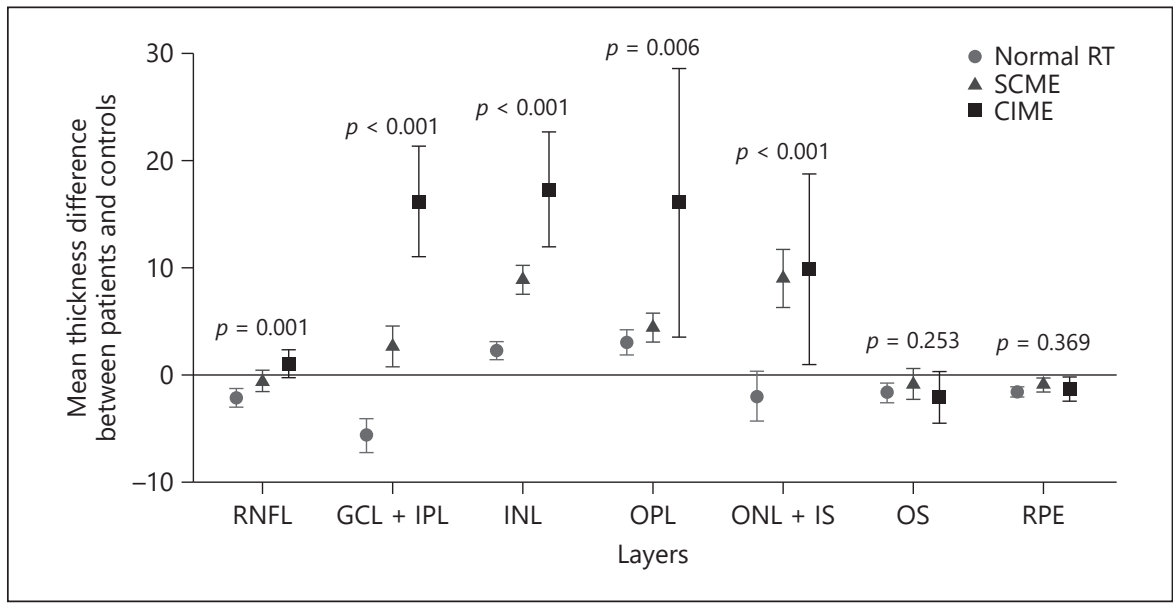

Fig. 2. Eyes with macular edema, either subclinical (SCME) or central-involved (CIME), by ETDRS group.

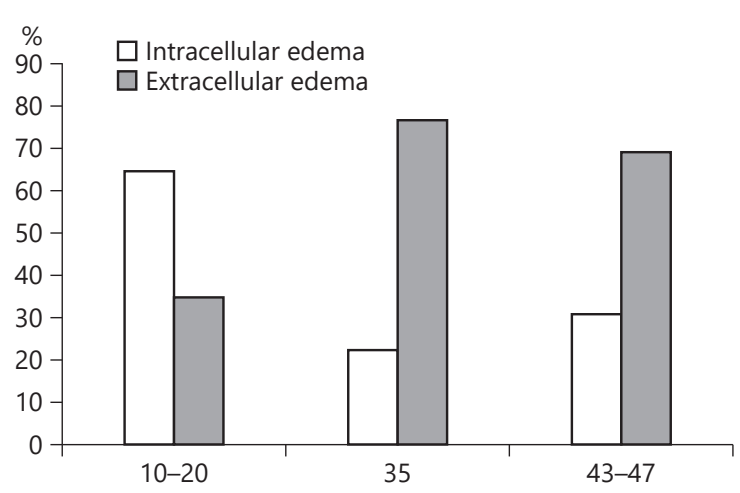

Retinal Vessel Density (Ischemia)

Increased LOR ratios, indicative of an abnormal collection of extracellular fluid, showed a significant correlation with decreased retinal vessel density in the SRP and DRP. In eyes with increased LOR ratio, 13 (38.2\%) presented possible decreases in vessel density in the SRP and definite in 10 (29.4\%; $p<0.001)$. In the DRP, $4(11.8 \%)$ presented possible decreases and definite in 10 (29.4\%; $p=0.038)$.

\section{Discussion}

Macular edema is identified by the increased thickening of the retina. It can occur in a variety of retinal diseases and is the result of an excess of fluid within the retinal tissue [18]. This accumulation of the fluid may be extracellular, intracellular or a combination of both [18]. 


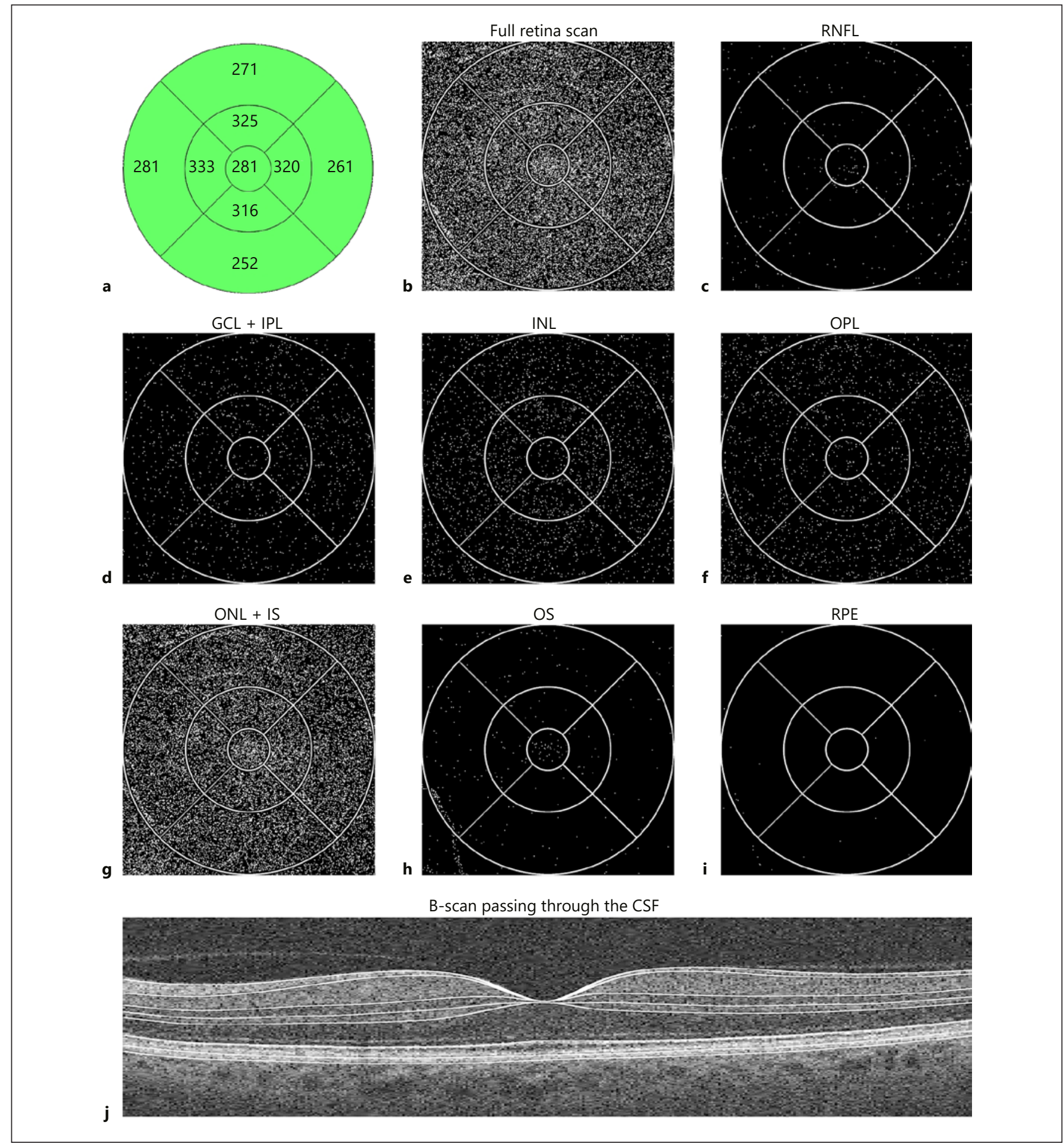

Fig. 3. Intracellular thickening (normal range of LOR ratios) in a ETDRS 10-20 level eye of a male diabetic patient. (a) OCT retinal thickness map (SCME); (b) OCT-Leakage map for the full retina; (c-i) OCT-Leakage maps for the retinal nerve fiber layer, ganglion cell and inner plexiform layers, INL, OPL, ONL+IS, outer segment, retinal pigment epithelium; (j) A B-Scan passing through the cen- tral subfield showing the segmented retinal layer interfaces in white. RNFL, retinal nerve fiber layer; GCL+IPL, ganglion cell layer and inner plexiform layer; INL, inner nuclear layer; OPL, outer plexiform layer; ONL+IS, outer nuclear layer and inner segment; OS, outer segment; RPE, retinal pigment epithelium; CSF, central subfield. 


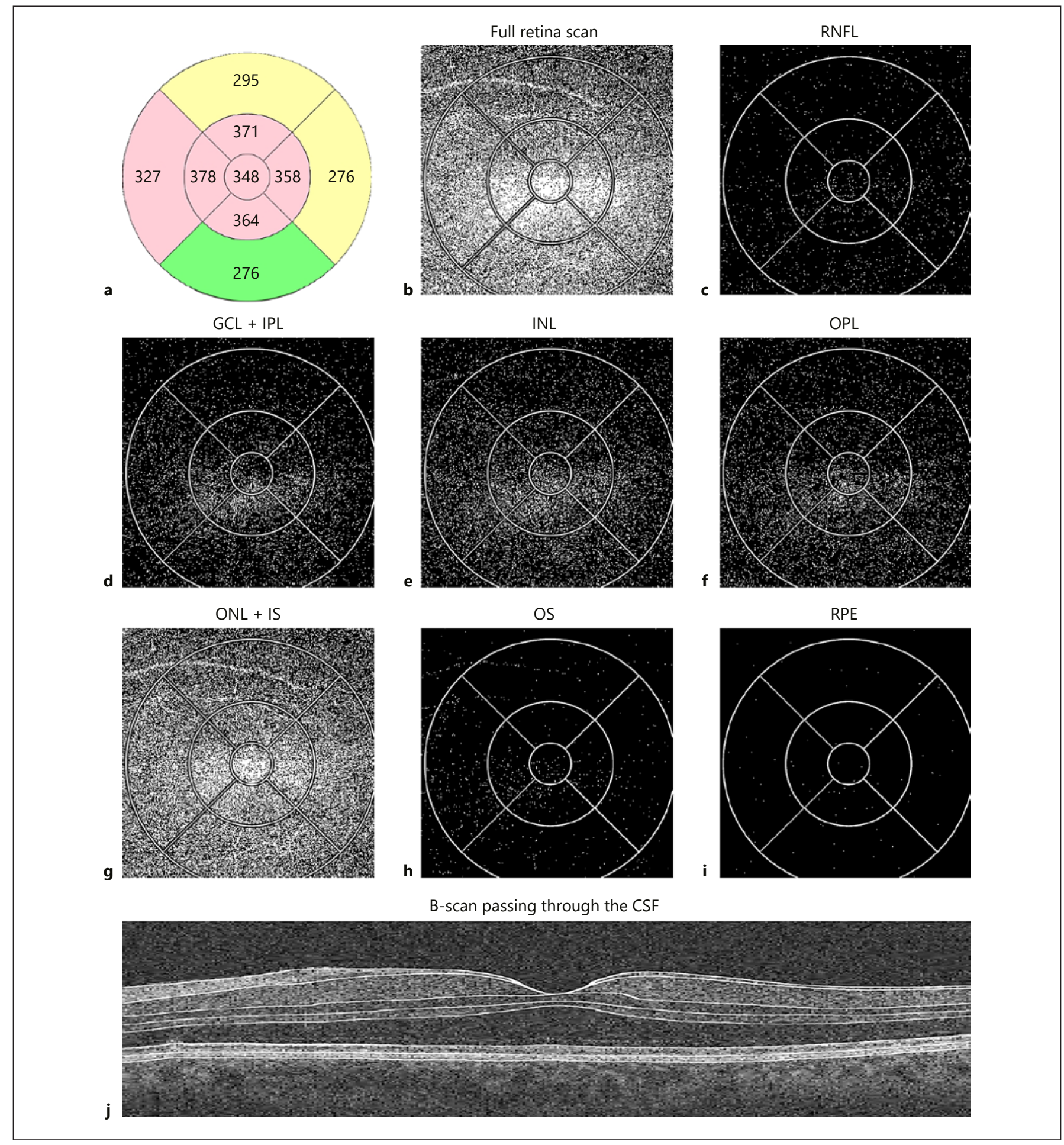

Fig. 4. Example showing extracellular edema (increased LOR ratios) in a ETDRS 35 level eye of a male diabetic patient. (a) OCT retinal thickness map (CIME); (b) OCT-Leakage map for the full retina; (c-i) OCT-Leakage maps for the retinal nerve fiber layer, ganglion cell and inner plexiform layers, INL, OPL, ONL+IS, outer segment, retinal pigment epithelium; (j) A B-Scan passing through the central subfield showing the segmented retinal layer interfaces in white. RNFL, retinal nerve fiber layer; GCL+IPL, ganglion cell layer and inner plexiform layer; INL, inner nuclear layer; OPL, outer plexiform layer; ONL+IS, outer nuclear layer and inner segment; OS, outer segment; RPE, retinal pigment epithelium; CSF, central subfield. 
The classification of retinal edema may follow the classification proposed by Klatzo [19] for brain edema, who divided it as cytotoxic or vasogenic. Cytotoxic referred to edemas that are predominantly intracellular and are associated with primarily neuronal or glial toxic damage. Vasogenic is the edema resulting from vascular damage and subsequent alteration of the blood-retinal barrier, which is mainly associated with an abnormal accumulation of extracellular fluid.

The normal retina, like the brain, has a functional extracellular space in the order of $24 \%$ of the total tissue [ 20 , 21]. The distribution of extracellular fluid varies between the different layers of the retina and appears to correspond to the paravascular fluid pathways that have been recently proposed to function as a glymphatic system [22].

Identification of extracellular fluid location in the retina was achieved by mapping the sites of LOR ratios, using an OCT-based method recently described by our group, designated OCT-Leakage [16].

The present study shows that macular edema can occur in approximately one third of diabetic eyes with minimal, mild or moderate retinopathy. The prevalence of diabetic macular edema does not follow the increase in severity of the retinopathy, as identified by ETDRS grading, appearing to occur independently of the severity of the retinopathy.

This study also shows that there are differences in the layer location and type of edema in different patients and in different ETDRS severity levels. In mild (ETDRS grade 35) and moderate (ETDRS grades $43-$ 47) retinopathy, the LOR ratios are clearly increased and the edema appears to be predominantly extracellular. When there is only minimal retinopathy (ETDRS group 10-20), the edema is located predominantly in the INL without corresponding increase in the LOR ratios suggesting that at least initially the retinal edema is intracellular and associated with an initial swelling of the retinal cells [6].

When CIME is identified by reaching high threshold thickness values, the edema is mainly extracellular with good correspondence between RT and LOR values. This increase in extracellular fluid is predominantly located in the INL, spreading to neighboring layers of the retina.

In a recent report by our group, we have shown that eyes with the same ETDRS retinopathy grading from different diabetic patients show different prevalence of the main disease pathways, neurodegeneration, edema, and ischemia. These main disease pathways do not occur in the same eyes suggesting that different disease pathways predominate in different patients, particularly in the most initial stages of retinopathy [17]. These findings reinforce the concept of different phenotypes of progression previously reported $[23,24]$.

One limitation of this study is its use of automated retinal layer segmentation analyses. These, however, were performed in retinas that remained structurally preserved with no evidence of cystoid changes, and they were reviewed by a masked grader.

These findings may have implications in our understanding of DR progression, its initial pathology and in the development of retinal edema. Our findings suggest that the edema may be initiated as a result of diabetic cytotoxic damage of Müller cells or other neuronal cells. Later, as the disease progresses, a vascular response predominates with breakdown of the blood-retinal barrier and increase in extracellular fluid.

\section{Disclosure Statement}

No potential conflicts of interest relevant to this article were reported.

\section{References}

1 Klein R, Klein BE, Moss SE, Davis MD, DeMets DL. The Wisconsin epidemiologic study of diabetic retinopathy. II. Prevalence and risk of diabetic retinopathy when age at diagnosis is less than 30 years. Arch Ophthalmol. 1984 Apr;102(4):520-6.

2 Krzystolik MG, Strauber SF, Aiello LP, Beck RW, Berger BB, Bressler NM, et al.; Diabetic Retinopathy Clinical Research Network. Reproducibility of macular thickness and volume using Zeiss optical coherence tomography in patients with diabetic macular edema. Ophthalmology. 2007 Aug;114(8):1520-5.
3 Brown JC, Solomon SD, Bressler SB, Schachat AP, DiBernardo C, Bressler NM. Detection of diabetic foveal edema: contact lens biomicroscopy compared with optical coherence tomography. Arch Ophthalmol. 2004 Mar; 122(3):330-5

4 Browning DJ, Fraser CM. The predictive value of patient and eye characteristics on the course of subclinical diabetic macular edema. Am J Ophthalmol. 2008 Jan;145(1):149-54.

5 Bressler NM, Miller KM, Beck RW, Bressler SB, Glassman AR, Kitchens JW, et al.; Diabetic Retinopathy Clinical Research Network. Ob- servational study of subclinical diabetic macular edema. Eye (Lond). 2012 Jun;26(6):833-40.

6 Bandello F, Tejerina AN, Vujosevic S, Varano M, Egan C, Sivaprasad S, et al.; EVICR.net. Retinal layer location of increased retinal thickness in eyes with subclinical and clinical macular edema in diabetes type 2 . Ophthalmic Res. 2015;54(3):112-7.

7 Vujosevic S, Midena E. Retinal layers changes in human preclinical and early clinical diabetic retinopathy support early retinal neuronal and Müller cells alterations. J Diabetes Res. 2013;2013:905058. 
8 Farinha C, Santos T, Marques IP, Marques JP, Ribeiro L, Figueira J, et al. OCT-Leakage Mapping: A New Automated Method of OCT Data Analysis to Identify and Locate Abnormal Fluid in Retinal Edema. Ophthalmol Retina. 2017 Nov; 1(6):486-96.

9 Santos AR, Alves D, Santos T, Figueira J, Silva R, Cunha-Vaz JG. Measurements of Retinal Fluid by OCT Leakage in Diabetic Macular Edema: A Biomarker of Visual Acuity Response to Treatment. Retina. 2019 Jan;39(1): $52-60$.

10 Santos T, Correia A, Neves CA, Schwartz C, Miranda T, Santos AR, et al. Feasibility of automated interface segmentation of Cirrus HD-OCT data in normal and mild non proliferative diabetic retinopathy eyes. Invest Ophthalmol Vis Sci. 2015 Jun;56(7):5953-5953.

11 Santos AR, Gomes SC, Figueira J, Nunes S, Lobo CL, Cunha-Vaz JG. Degree of decrease in central retinal thickness predicts visual acuity response to intravitreal ranibizumab in diabetic macular edema. Ophthalmologica. 2014;231(1):16-22.

12 Kim BY, Smith SD, Kaiser PK. Optical coherence tomographic patterns of diabetic macular edema. Am J Ophthalmol. 2006 Sep; 142(3):405-12.

13 Santos AR, Costa MÂ, Schwartz C, Alves D, Figueira J, Silva R, et al. OCT Baseline Predictors for Initial BCVA Response to Intravitreal
anti-VEGF Treatment in Eyes with Diabetic Macular Edema. The CHARTRES study. Retina. 2018 Jun;38(6):1110-9.

14 Sun JK, Lin MM, Lammer J, Prager S, Sarangi R, Silva PS, et al. Disorganization of the retinal inner layers as a predictor of visual acuity in eyes with center-involved diabetic macular edema. JAMA Ophthalmol. 2014 Nov;132(11):1309-16.; Epub ahead of print.

15 Friedman SM, Almukhtar TH, Baker CW, Glassman AR, Elman MJ, Bressler NM, et al.; Diabetic Retinopathy Clinical Research Network. Topical nepafenec in eyes with noncentral diabetic macular edema. Retina. 2015 May;35(5):944-56.

16 Cunha-Vaz J, Santos T, Ribeiro L, Alves D, Marques I, Goldberg M. OCT-leakage: A new method to identify and locate abnormal fluid accumulation in diabetic retinal edema. Invest Ophthalmol Vis Sci. 2016 Dec;57(15): 6776-83.

17 Marques IP, Alves D, Santos T, Mendes L, Santos AR, Lobo C, et al. Multimodal Imaging of the Initial Stages of Diabetic Retinopathy. Different Disease Pathways in Different Patients. Diabetes. Diabetes. 2019 Mar;68(3): 648-653.

18 Henkind P, Bellhorn R, Schall B. Retinal Edema: Postulated Mechanism(s). In: Cunha-Vaz JG, editor. The Blood-Retinal
Barriers. Boston, MA: Springer US; 1980. pp. 251-68.

19 Klatzo I. Presidental address. Neuropathological aspects of brain edema. J Neuropathol Exp Neurol. 1967 Jan;26(1):1-14.

20 Smelser GK, Ishikawa T, Pei YF. Electron microscopic studies of intra-retinal spaces: diffusion of particulate materials. In: Rohen JW, editor. Structure of the Eye, II Symp. Stuttgart: Schattauer-Verlag; 1965. p. 109-21.

21 Cheek DB, Holt AB. A review: extracellular volume in the brain - the relevance of the chloride space. Pediatr Res. 1978 May;12(5):635-45.

22 Iliff JJ, Wang M, Liao Y, Plogg BA, Peng W, Gundersen GA, et al. A paravascular pathway facilitates CSF flow through the brain parenchyma and the clearance of interstitial solutes, including amyloid $\beta$. Sci Transl Med. 2012 Aug;4(147):147ra111.

23 Santos AR, Ribeiro L, Bandello F, Lattanzio R, Egan C, Frydkjaer-Olsen U, et al.; European Consortium for the Early Treatment of Diabetic Retinopathy (EUROCONDOR). Functional and structural findings of neurodegeneration in early stages of diabetic retinopathy: cross-sectional analyses of baseline data of the EUROCONDOR project. Diabetes. 2017 Sep; 66(9):2503-10.

24 Cunha-Vaz J, Ribeiro L, Lobo C. Phenotypes and biomarkers of diabetic retinopathy. Prog Retin Eye Res. 2014 Jul;41:90-111. 